\title{
Fuzzy logic and neural networks - Applications to analytical chemistry
}

\author{
Matthias Ottol, Tatjana George ${ }^{1}$, Claudia Schierle ${ }^{1}$ and \\ Wolfhard Wegscheider \\ 1 Bergakademie Freiberg, Institute for Analytical Chemistry, \\ Leipziger Str. 29, 9200 Freiberg/Sachsen, F.R.G. \\ 2 Graz University of Technology, Institute for Analytical \\ Chemistry, Micro- and Radiochemistry, Technikerstr. 4, \\ A- 8010 Graz, Austria
}

\begin{abstract}
A general overview about the usefulness of the theory of fuzzy sets for solving problems in the field of data analysis and analytical reasoning as well as for using neural networks for knowledge processing in analytical chemistry is given. Recent results of the development of intelsystems for automated qualitative analysis in $X$-ray fluorescence analysis and in ICP-atomic emission spectrosocopy are discussed.
\end{abstract}

\section{INTRODUCTION}

There is an increasing interest in Analytical Chemistry in developing intelligent systems that approach human analytical reasoning as close as possible and that enable analytical knowledge to be acquired automatically.

Besides artificial intelligent techniques based on symbolic programming these goals can be pursued by applying the theory of fuzzy sets for matching the analyst's way of reasoning and by neural networks to automate knowledge acquisition and interpretation. Although there is intensive research going on to combine techniques of fuzzy sets and neural networks [1] the present paper will handle these two methods separately.

It can be shown that basic operations of the theory of fuzzy sets can be used in analytical chemistry to solve problems, such as library searching, depth profile comparison or calibration with errors in signals and concentrations. The major area of applications for fuzzy logic, however, concerns the development of expert or intelligent systems, e.g. for spectra interpretation or reasoning in chemical data bases. An example for fuzzy reasoning is given here for rule building in an expert system for automated qualitative analysis with $\mathrm{X}$-ray fluorescence spectroscopy.

Neural networks are studied in analytical chemistry with respect to pattern recognition, modeling and prediction, e.g. in multicomponent analysis or process control, to classification, clustering or pattern association. Based on the latter technique recent advances are reported for developing an expert system for qualitative ICPatomic emission spectroscopy. 


\section{FUZZY LOGIC}

The theory of fuzzy sets was introduced by L,A. Zadeh [2] as an extension of classical set theory and Boolean logic. With common (crisp) set theory, an item is either a member of a set or it is not. For example an element is either a 'metal' or a 'nonmetal'. In fuzzy set theory degrees of set membership allow to define a transition from membership to nonmembership. Thus, in our example the theory provides a tool to describe an element being both a 'metal' and a 'non-metal' to a certain degree.

Mathematically the concept of gradual membership of an item to a set can be formulated by a characteristic function, the membership function (m.f.), $m(x)$, which assigns a value of 1 to every element $x$ of the total population $X(x \in X)$ that is a member of a subset $A$ and a membership value of 0 to elements that are not members of $A$ :

$$
m(x)=\left\{\begin{array}{llll}
1 & \text { if } & x \in A & x \\
0 & \text { if } & x \notin A & X
\end{array}\right.
$$

Fuzzy sets are then characterized by m.f.'s that allow values between 0 and 1 , for example of the type:

$$
m(x)=\left[1-\exp \left[-c(x-a)^{2}\right]\right.
$$

where a and $c$ are constants that must be positive numbers (Fig.1). Membership functions can be based on experimental data, on experts knowledge or subjective aspects or they can be implemented by generating rules, e.g. in knowledge based systems.

The operations on fuzzy sets can be understood as an extension of classical set theory.

In Figure 2 fuzzy set operations for the union between two sets A and $B(A \cup B)$, the intersection $(A \cap B)$, the complement $\bar{A}$ of set $A$ and the cardinality or power of a set $A$ are demonstrated graphically.

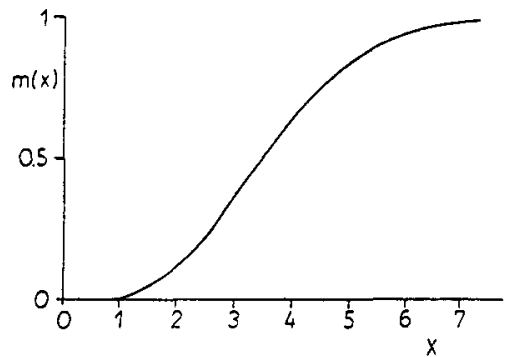

Fig. 1. Membership function according to equ. (1), describing a fuzzy set "high".
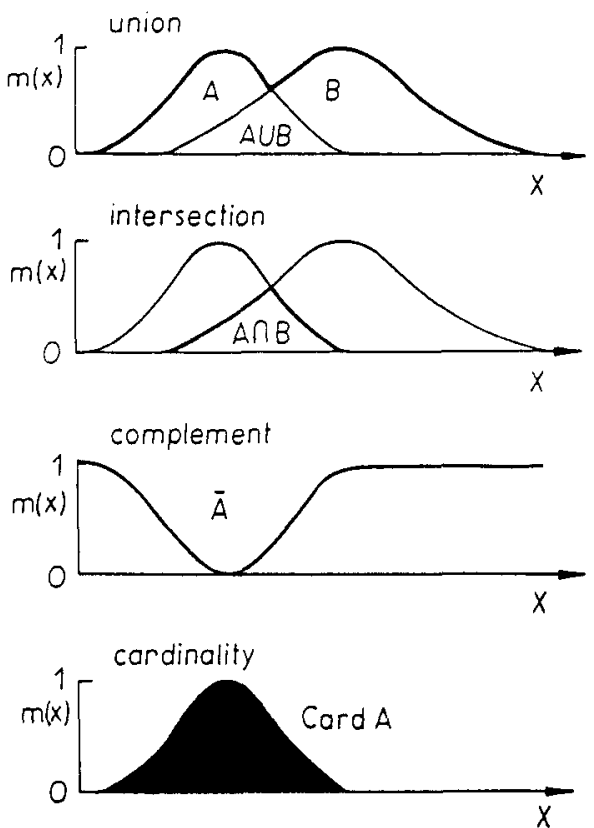

Fig. 2. Operations on fuzzy sets 
With these simple operations analytical problems have been solved with respect to multi-criteria decision making [3] or pattern matching in spectroscopy or chromatography [4].

Recently we applied these fundamental fuzzy set operations in an interpretation system for automated qualitative analysis in $X-r a y$ fluorescence spectroscopy [5]. The measured X-ray fluorescence spectrum is transfered to a line spectrum by using the 2nd derivative computed by a Savitzky-Golay digital filter and the resulting lines are fuzzified according to their variability (Fig. $3)$.

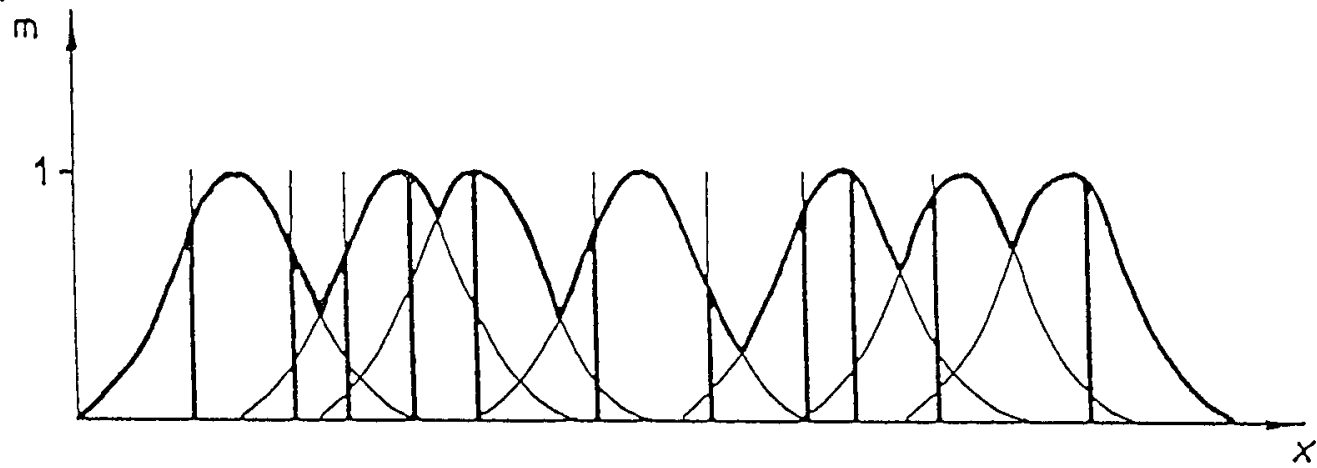

Fig. 3. Comparison of a fuzzified sample spectrum with a crisp candidate reference spectrum by fuzzy intersection

In the next step the membership funtions have to be unified as demonstrated in Fig. 3 by bold lines covering the membership funtions. Comparison of the measured spectrum to the library spectra of the elements from sodium (atomic number 11) to uranium (atomic number 92) is performed by fuzzy intersection. In this approach the library spectrum is assumed to be crisp. As the result of intersection discrete membership values are obtained that are aggregated by calculating the relative cardinality, i.e. the sum of the membership values normalized to the number of lines in the candidate reference spectrum.

To aid analytical reasoning the theory of fuzzy sets is used in the sense of fuzzy logic and approximate reasoning. Fuzzy logical operations are defined for all the logical connectives known from classical (Boolean) logic, such as AND, OR, NOT or the implication. In most applications a fuzzy logical AND is defined by intersection, a fuzzy OR by the union, the NOT by the fuzzy complement and an implication (IF $V=A$ THEN $U=B$ ) by the membership funtion over the cartesian product $A \times B$ represented by the minimum between the m.f.'s of $A$ and $B$.

Fuzzy logic has been mainly applied until now in the context of fuzzy control. In chemistry this idea has been used by Yamada [6] to control fermentation of coenzyme Q10 based on monitoring cell mass with a turbidimetric sensor. In this way the very complex process that cannot be described by a mathematical model is controlled by some rules of thumb specified by fuzzy sets.

In the above mentioned example of X-ray fluorescence analysis fuzzy logic can be used to specify the following types of rules:

- The intensity ratio of $K_{a}$ and $K_{B}-1$ ines should be "about 5 ".

- For elements with atomic numbers less than 20 the detection probability of $\mathrm{K}_{B}$-lines is "decreased heavily".

- Resolution of LB 1 and LB 5 lines "decreases with increasing atomic numbers". 
These rules are formalized in the usual manner as implications, e.g.

IF the line pair is $K_{a}, K_{B}$ THEN intesity ratio is "about 5 "

The data "about 5" is represented here as a fuzzy number and the degree of membership of a measured intensity ratio is infered by applying Zadeh's compositional rule of inference [7].

Different schemes of reasoning can be applied, for example the theory of approximate reasoning as further developed by Yager [8].

Approximate reasoning has been already explored in analytical chemistry for reasoning about missing data/information in a data base on pH-indicators [9] and is used at present for building an interpretation system in IR-spectroscopy.

The concept of fuzzy theory is also used for data analysis in analytical chemistry. A difference to statistical data analysis can be understood as follows: a statistical (probabilistic) approach describes the set of potential outcomes on the basis of a certain distribution. The observation results is vague only before experimentation but is a determined object after sampling. In a fuzzy-set theoretic (possibilistic) approach the concrete observation is available only with a certain vagueness either due to the uncertainty of the data themselves or due to our description of the observed object by linguistic expressions.

Because both types of observations can be specified as fuzzy sets data analysis can be carried out independent on the observation type. Up to now fuzzy data analysis has been applied to univariate and multivariate modeling [4], to pattern recognition problems [1012] or to clustering of data [13]. For example, grouping of malt samples analysed for 9 physico-chemical parameters was performed on the basis of fuzzy clustering [13]. The advantage of fuzzy methods of clustering over conventional crisp cluster

algorithms is the feasibility to assign a single object to more than one cluster simultaneously.

By means of pattern recognition gasoline samples analysed by capillary gas chromatography can be matched to typical reference chromatograms of fuels in a library [14].

\section{NEURAL NETWORKS}

Neural networks or parallel distributed processing is an alternative to sequential processing of knowledge as known from symbolic programming. In analogy to the human brain artificial neural networks consist of single units (neurons) that are interconnected by the so-called synapses. The typical network has layers of input and output units that are either connected directly or they are linked through one or several hidden layers (Fig. 4).

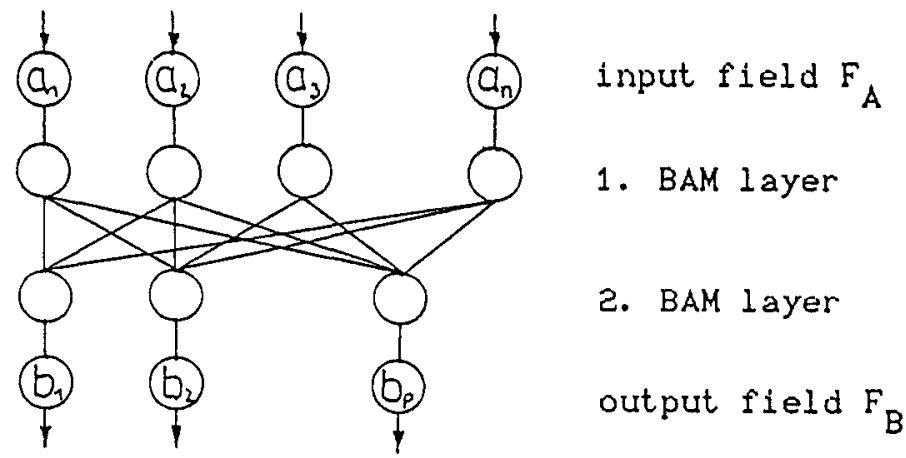

Fig. 4. The Bidirectional Associative Memory (BAM) as an example for a neural network with two hidden layers. 
Each unit sends its numeric output modified by inhibitory or excitatory weights (also called transition coefficients) to another unit so that the output of a unit is the input to another or to many other units. Usually, one single unit multiplies the inputs by the individual weights and sums them up. The resulting value is the activation value of the unit which is often modified by applying an appropriate transformation function. The activation value is then proceeded to other units by applying the appropriate weight.

In contrast to classical AI-techniques where knowledge is represented explicitly in form of rules, with neural networks the knowledge is implicitly stored in the weights. By presenting a certain input pattern to the network the correct output units will be activated.

Examples for applying neural networks in analytial chemistry are known for interpretation of IR spectra [14], for classification of ${ }^{1} \mathrm{H} \mathrm{NMR-} \mathrm{[15],} \mathrm{IR[16]} \mathrm{or} \mathrm{mass} \mathrm{spectra} \mathrm{[17,18],} \mathrm{for} \mathrm{identification} \mathrm{of}$ UV-spectra [19] or of odors based in a quartz-resonator sensor array [20], for multicomponent analysis in the near infrared range [21] or with ion-selective electrodes [22].

These applications are based on the main feature of neural networks to allow (hetero-)associations to be learned between an input pattern, e.g. a spectrum, and an output pattern, that might contain structural or concentration information. Sometimes these associations are formulated as classification schemes where the output is related to the membership to a certain class, e.g. the class of all aliphatic alcohols.

Neural networks can also be used to group data in the sense of clustering or regularity detection, e.g. molecular spectra of chemically similar compounds should group into related clusters.

The performance of the neural network heavily depends on the network architecture and on the implemented learning algorithm. Almost all of the applications use multi-layer perceptrons, i.e. networks with one or several hidden layers and the back-propagation algorithm as the learning strategy.

An example of neural network application of a different approach is given here with the Bidirectional Associative Memory (BAM) [23] for interpretation of ICP- atomic emission spectra.

The BAM consists of an input, an output and two BAM layers interconnected as given in Fig. 4 .

The ICP-spectrum is presented to the input layer and the output layer represents the appropriately encoded elements. Association of single elements with their related spectra is carried out in the encoding (training) phase. A feedback between the two BAM layers is possible so that imformation can flow in both directions. If for analysis of an unkown spectrum the sample spectrum is shown to the BAM input he output neurons representing the present elements should fire.

For interpretation of ICP-spectra the original BAM was modified with respect to the neuron aggregation in order to account for the different importance of prominent and nonprominent lines. Aggregation is performed with the ordered weighted averaging (OWA) operator introduced by Yager [24] in a way that for an element at least two prominent lines and most of the remaining prominent lines should be found. By combining the qualitative results of the neural network output with a least squares methods based on the known sensitivities at the different lines a semi-quantitative analysis can be carried. 
Acknowledgement : The financial support by the Deutsche Forschungsgemeinschaft is gratefully acknowledged.

\section{REFERENCES}

1. Proceedings of the International Conference on Fuzzy Logic \& Neural Networks, Iizuka (Japan), July 22-24 (1990).

2. L.A. Zadeh, Inf. Control 8, 338-353 (1965).

3. M. Otto, Chemometrics Intelligent Lab. Sys. 4, 101-120 (1988).

4. H. Bandemer and M. Otto, Mikrochim. Acta [Wien] II, 93-124 (1986).

5. T. George, M. Otto and W. Wegscheider, in preparation.

6. Y. Yamada, S. Murayama and S. Shiomi, J. Chem. Eng. of Japan 24, 94-99 (1991).

7. L.A. Zadeh, in J.E. Hayes, D. Michie and L.I. Kulich (Eds.), Machine Intelligence 9, Wiley, New York, 149-194 (1979).

8. R.R. Yager, Artificial Intell. 31, 99-112 (1987).

9. M. Otto and R.R. Yager, in G. Gauglitz (Ed.), SoftwareEntwicklungen in der Chemie 3, Springer-Verlag, Berlin Heidelberg, 229-245 (1989).

10. T. Blaffert, Anal. Chim. Acta 161, 135-148 (1984).

11. M. Otto and H. Bandemer, Anal. Chim. Acta 184, 21-31 (1986).

12. E. Stottmeister, H. Hermann, P. Hendel, D. Feiler, M. Nagel, and H. J. Dobberkau, Fresenius Zeitschrift f. Anal. Chemie 327, 709-714 (1987).

13. T. Jacobson, K. Kolset and N.B. Vogt, Mikrochim. Acta [Wien] II, $125-138$ (1986).

14. E.W. Robb and M.E. Munk, Mikrochim. Acta [Wien] I, 131-155 (1990).

15. J.U. Thomsen and B. Meyer, J.Magn. Reson. 84, 212-217 (1989).

16. J. Zupan, Anal. Chim. Acta 235, 53-63 (1990).

17. M. Bos, A. Bos and W. E. Van der Linden, Anal. Chim. Acta 233, 31-39 (1990).

18. B. Curry and D.E. Rumelhart, Tetrahedron Comp. Method., in press (1991).

19. M. Otto and U. Hörchner, in J. Gasteiger (Ed.), Software Development in Chemistry 4 , Springer-Verlag, Berlin Heidelberg, 377-384 (1990).

20. T. Nakamoto, K. Fukunishi and T. Moriizumi, Sensors and Actuators B1, 473-476 (1990).

21. J. Long, V.G. Gregorion and P.J. Gamberline, Anal. Chem. 62, 1791-1797 (1990).

22. M. Bos, A. Bos and W.E. Van der Linden, Anal. Chim. Acta 233, $31-39(1990)$

23. B. Kosko, Appl. Optics 26, 4947-4960 (1987).

24. R.R. Yager, IEEE Trans. Syst. Man Cybern. 18, 183-190 (1988). 